\title{
Systematic Review \\ Efficacy and Safety of Tirzepatide in Patients with Type 2 Diabetes Mellitus: A Systematic Review and Meta-Analysis of Randomized Phase II/III Trials
}

\author{
Akshaya Srikanth Bhagavathula ${ }^{1, *,+}$, Kota Vidyasagar ${ }^{2,+}$ (D) and Wubshet Tesfaye ${ }^{3,+}$ (D) \\ 1 Department of Social and Clinical Pharmacy, Faculty of Pharmacy in Hradec Králové, Charles University, \\ 50115 Hradec Králové, Czech Republic \\ 2 University College of Pharmaceutical Sciences, Kakatiya University, Warangal 506009, India; \\ vidyasagarkota2@gmail.com \\ 3 Health Research Institute, University of Canberra, Canberra, ACT 2617, Australia; \\ wubshet.tesfaye@canberra.edu.au \\ * Correspondence: akshaypharmd@gmail.com; Tel.: +420-776-444-393 \\ + These authors contributed equally to this work.
}

\section{check for}

updates

Citation: Bhagavathula, A.S.; Vidyasagar, K.; Tesfaye, W. Efficacy and Safety of Tirzepatide in Patients with Type 2 Diabetes Mellitus: A Systematic Review and Meta-Analysis of Randomized Phase II/III Trials. Pharmaceuticals 2021, 14, 991. https://doi.org/10.3390/ph14100991

Academic Editors: Magdalena Kotańska, Amélia Pilar Rauter, Małgorzata Szafarz and Monika Kubacka

Received: 9 September 2021

Accepted: 25 September 2021

Published: 28 September 2021

Publisher's Note: MDPI stays neutral with regard to jurisdictional claims in published maps and institutional affiliations.

Copyright: (c) 2021 by the authors. Licensee MDPI, Basel, Switzerland. This article is an open access article distributed under the terms and conditions of the Creative Commons Attribution (CC BY) license (https:/ / creativecommons.org/licenses/by/ $4.0 /)$.

\begin{abstract}
Tirzepatide is a novel once-a-week dual glucose-dependent insulinotropic polypeptide and glucagon-like peptide-1 receptor agonist, currently under trial to assess glycemic efficacy and safety in people with type 2 diabetes. A systematic review and meta-analysis were conducted to investigate the efficacy of tirzepatide on glycated hemoglobin (HbA1c, \%), fasting serum glucose (mg/dL), and body weight $(\mathrm{kg})$ in patients with uncontrolled type 2 diabetes (HbA1c $>7.0 \%)$. Mean changes for efficacy and proportions (safety) with corresponding 95\% confidence intervals (CIs) were used to provide pooled estimates. A total of four randomized controlled trials, comprising 2783 patients of whom 69.4\% ( $\mathrm{n}=1934)$ were treated with $5 \mathrm{mg}(\mathrm{n}=646), 10 \mathrm{mg}(\mathrm{n}=641)$, or $15 \mathrm{mg}(\mathrm{n}=647)$ of tirzepatide, were compared to the placebo $(n=192)$ or the selective GLP-1 receptor agonist $(n=523)$. The pooled analysis showed that tirzepatide treatment resulted in a greater lowering of the $\mathrm{HbA} 1 \mathrm{c}$ (-1.94\%, 95\% CI: -2.02 to -1.87$)$, fasting serum glucose (-54.72 mg/dL, 95\% CI: -62.05 to -47.39$)$, and body weight $(-8.47,95 \%$ CI: -9.66 to -7.27$)$. We also found that improvement in the HbA1c levels was still maintained at weeks 26 and 40 from the long-term trials. As for safety, only $3 \%$ experienced hypoglycemia, and 4\% (95\% CI: 2 to 6) experienced serious adverse events, while the discontinuation of therapy percentage was 7\% (95\% CI: 5 to 8). Tirzepatide significantly improved glycemic control and body weight and had an acceptable safety profile, indicating that it is an effective therapeutic option for glucose-lowering in patients with type 2 diabetes mellitus.
\end{abstract}

Keywords: LY3298176; tirzepatide; diabetes; glucose; randomized controlled trial; efficacy

\section{Introduction}

Diabetes mellitus affects over 463 million people globally as of 2019, with this number projected to grow to 700 million by 2045 [1]. The well-recognized microvascular and macrovascular damages associated with type 2 diabetes mellitus (T2DM) make it among the leading causes of serious complications, including blindness, kidney failure, and cardiovascular complications, and associated mortality [2,3]. Optimal management of T2DM is required to effectively prevent the occurrence of diabetes-related complications or delay the deterioration of these complications if they are already present [4]. A range of drug classes with different modes of action have been in use for the management of diabetes and/or its major clinical sequelae.

While the various classes of T2DM medications have their own benefits, ideal treatments would be those that are effective in reducing blood glucose levels, are associated with less risk of hypoglycemia and weight gain, and have added benefits for cardiovascular or renal outcomes [5]. Some of the newly emerging treatments used for T2DM, such as 
glucagon-like peptide-1 (GLP-1) receptor agonists and the sodium-glucose cotransporter-2 inhibitors, have, for example, showed promising outcomes in recent years in terms of providing additional cardiorenal benefits in addition to controlling diabetes [6].

GLP-1 agonists, which include short-acting or long-acting agents, have been associated with improved glycemic control and cardiovascular outcomes, as well as body weight reduction in people with T2DM [7]. The other incretin hormone, glucose-dependent insulinotropic polypeptide (GIP), despite its similarity with GLP-1 and its receptors, is devoid of effects like the inhibition of appetite and food intake, which is seen with the use of GLP-1 [8]. Therefore, combining the GLP-1 agonists with other agents that act on glucosedependent insulinotropic polypeptide (GIP) receptors has been hypothesized to produce a more effective control of glycemia along with a significant weight reduction effect [9]. Tirzepatide is a novel antidiabetic treatment recently developed and has demonstrated favorable body weight, cardiovascular, and renal-related outcomes [10]. It acts as an agonist of both GLP-1 and GIP receptors, rendering it with an additive effect of producing a more pronounced insulin response than can be achieved through individual hormone administration $[5,11,12]$.

Early evidence revealed that tirzepatide is associated with a significantly positive outcome in controlling both blood glucose and body weight [8]. In light of additional emerging evidence based on phase II and III clinical trials investigating the effect of tirzepatide on glycemic and other added benefits, there is a need for more comprehensive data to consolidate the evidence and guide future research and potential clinical use. Therefore, this work aims to present a systematic review and meta-analysis of the literature published on phase II and III trials involving tirzepatide with a particular focus on its link with glycemic and body weight parameters.

\section{Materials and Methods}

\subsection{Search Strategy}

The systematic review and meta-analysis were executed in full accordance with the preferred reporting items for systematic review and meta-analyses guidelines [13]. A comprehensive literature search was conducted in the PubMed/Medline, Cochrane Library, Web of Science, and Scopus databases, which were searched for randomized controlled trials (RCTs) investigating the efficacy and safety of tirzepatide up to 30 July 2021 and published in the English language. The following medical subject heading terms and keywords were used for the database searches: (tirzepatide OR LY3298176 OR LY-3298176) AND (diabetes mellitus OR diabetes OR type 2 diabetes mellitus OR hyperglycaemia OR hyperglycemia OR hyperglycaemias OR hyperglycemias OR hyperglycemia s OR glucose OR glycated hemoglobin AND body weight). We also hand-searched the databases to identify additional relevant studies, references of relevant review articles, and conference proceedings.

\subsection{Study Selection}

Original studies that met the following criteria were included: (i) population: patients with uncontrolled T2DM, with no restriction on complications; (ii) were a phase II or III RCT with either a single-center or multicenter design; (iii) investigating the effect of tirzepatide on glucose parameters or body weight; (iv) intervention: tirzepatide at all doses (5 mg, 10 mg, $15 \mathrm{mg}$ ); (v) control: placebo or control; (vi) outcomes: efficacy and safety parameters related to the treatment; (vii) tested the efficacy and safety of tirzepatide in the short (12 weeks) and long term (26 and 40 weeks); and (viii) safety outcomes included all adverse events (AEs), hypoglycemic events, gastrointestinal events, and discontinuation of therapy.

The exclusion criteria included: (a) nonhuman studies and in vitro research, phase I clinical trials, case reports, editorials, conference proceedings, commentaries, expert opinions, reviews, studies without original data, non-RCTs, non-English publications, and duplicate publications; and (b) studies that lacked a control-treated group for comparison with tirzepatide. 


\subsection{Data Extraction}

Two authors independently performed the literature search. After the identification of eligible studies, the authors independently extracted data using standardized predefined forms for the first author's name; clinical trial registration number; year of publication; study sites; study design; baseline characteristics of the study population; age; the number of males; population; groups; duration of disease; baseline HbA1c; background glucose-lowering therapy; tirzepatide and comparator(s); dose/duration of tirzepatide; and outcomes measured. We extracted outcome data as arm-specific counts (i.e., number of participants, means differences, and standard error (or standard deviation) for continuous outcomes in patients with T2DM).

We also extracted data at the corresponding time point to appraise the $\mathrm{HbA1c}$ efficacy of tirzepatide in the short (week 12) and long term (weeks 26 and 40). Among the identified studies, the primary efficacy endpoint was the mean changes in $\mathrm{HbA1c}$, fasting serum glucose (FSG, mg/dL), and body weight $(\mathrm{kg})$ with tirzepatide at doses of $5 \mathrm{mg}, 10 \mathrm{mg}$, and $15 \mathrm{mg}$ monotherapy or add-on therapy to metformin in patients with T2DM. With regard to safety, we considered AEs, serious AEs, hypoglycemic events, gastrointestinal events, and AEs leading to discontinuation of treatment.

\subsection{Quality Assessment}

The risk of bias in the eligible RCTs was performed using the "Cochrane collaboration's tools for quality assessment of randomized controlled trials" [14]. The following domains were considered: (i) selection bias: random sequence generation and allocation concealment; (ii) blinding of outcome assessment (detection bias); (iii) incomplete outcome bias (attrition bias); (iv) selective reporting (reporting bias); and other bias (e.g., whether the baseline was comparable).

\subsection{Data Analysis}

All the analyses were performed using STATA MP 16.1 software (StataCorp LLC, College Station, TX, USA). Changes in continuous outcomes were calculated for each study arm by subtracting the value at baseline from the value after intervention. All the efficacy estimates were expressed as mean changes and $95 \%$ confidence interval (CI) from baseline. Standard deviations (SD) were calculated from the standard error or $95 \% \mathrm{CI}$, according to the Cochrane handbook for systematic review of interventions [15]. Safety estimates were presented as a pooled proportion with $95 \% \mathrm{CI}$.

The Higgins $I^{2}$ statistics and Cochran's $Q$ test were used to assess the potential statistical heterogeneity among trials. The meta-analysis was conducted using a fixed-effect model (using inverse-variance) or a random-effect model (DerSimonian-Laird method) based on low heterogeneity $(<50 \%)$ or high heterogeneity $(>50 \%)$. Publication bias was evaluated using funnel plots, Egger's regression asymmetry test, and Begg's correlation rank test [16]. A two-tailed $p$-value was considered significant below the level of 0.05 .

\section{Results}

\subsection{Study Characteristics}

From 242 identified records, we excluded nonhuman and irrelevant studies, leaving 16 reports for full-text assessment. After further selection (Figure 1), four unique RCTs fulfilled the inclusion criteria (Table 1) [17-20]. RCTs published between 2018 and 2021 included 2783 (range: 26-1490) participants with T2DM; three out of four studies were multinational RCTs. All the studies included T2DM patients controlled inadequately with diet and exercise alone or with stable metformin therapy. Baseline age and disease duration weighted means were $56.4 \pm 1.82$ and 8 years, respectively, $53 \%$ of the participants were male, and follow-up duration ranged from 12 to 40 weeks. Other characteristics of the RCTs, such as study details, drug- and outcome-specific data, are shown in Table 1. 
Identification of studies via databases
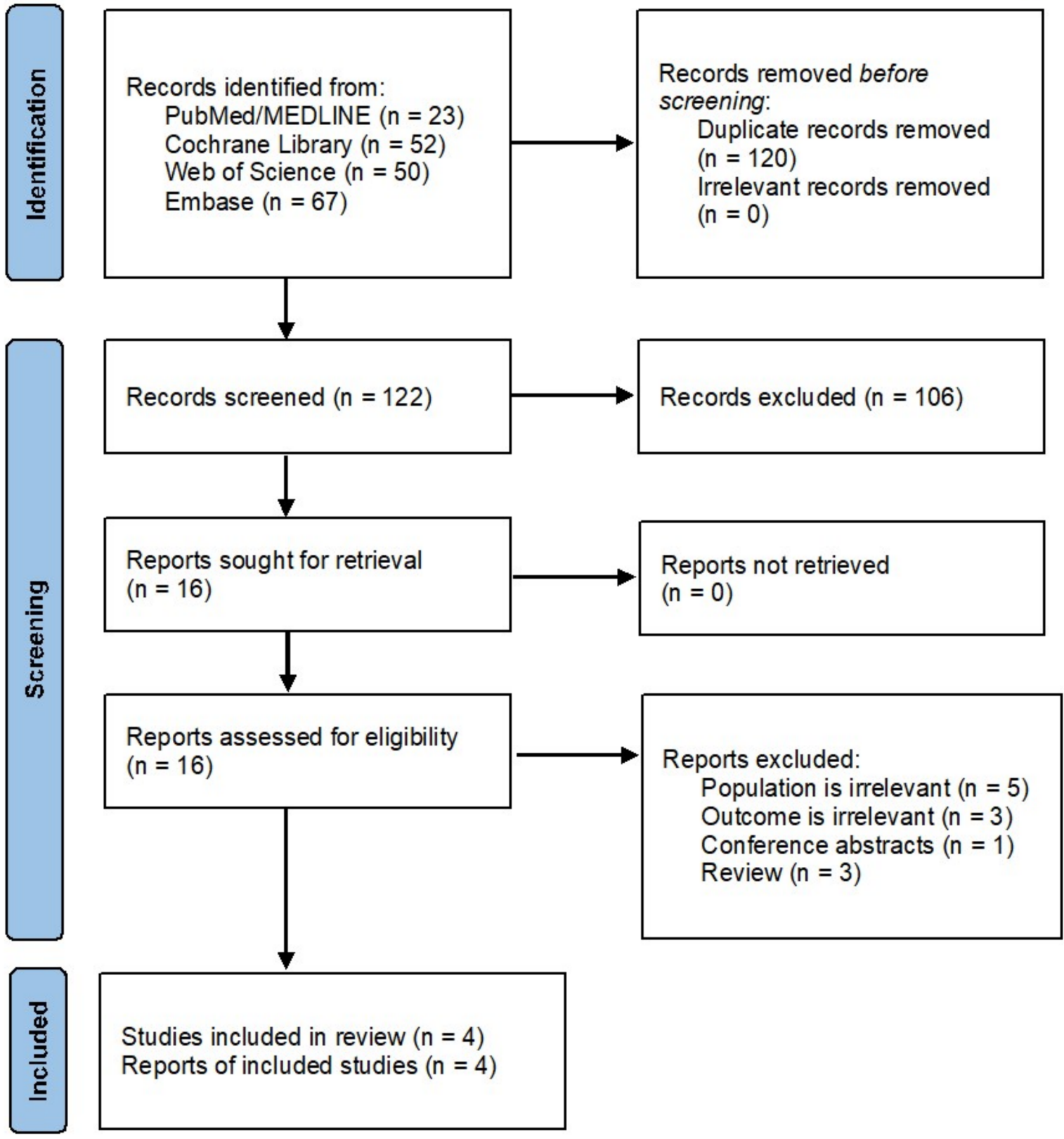

Studies included in review $(n=4)$

Reports of included studies $(n=4)$

Figure 1. PRISMA flowchart of literature search and study selection.

Overall, the risk of bias in eligible RCTs is shown in Figure 2. All included studies exhibit a low risk of selection bias, performance bias, detection bias, and reporting bias. The risk of bias in allocation concealment was generally unclear apart from the Rosenstock et al. [17] study. One study [20] has a high risk of bias because it did not provide details of blinding the participants and their outcome assessment. 
Table 1. Study characteristics of included studies.

\begin{tabular}{|c|c|c|c|c|c|c|c|c|c|c|c|c|c|}
\hline Author Name & NCTID & Location & Design & $\begin{array}{l}\text { Participants } \\
\text { Condition at } \\
\text { Baseline }\end{array}$ & $\begin{array}{c}\text { Disease } \\
\text { Duration } \\
\text { (years) }\end{array}$ & $\begin{array}{l}\text { Primary } \\
\text { Outcome }\end{array}$ & $\begin{array}{l}\text { Treatment } \\
\text { Duration } \\
\text { (weeks) }\end{array}$ & Intervention & $\begin{array}{c}\text { Patients on } \\
\text { Metformin } \\
\text { Therapy (\%) }\end{array}$ & $\begin{array}{c}\text { Number } \\
\text { Randomized }\end{array}$ & $\begin{array}{c}\text { Age } \\
\text { (Years) }\end{array}$ & $\begin{array}{l}\text { Male } \\
(\%)\end{array}$ & $\begin{array}{c}\text { Average } \\
\text { Change in } \\
\text { HbA1c (\%) } \\
\text { From Baseline }\end{array}$ \\
\hline \multirow{4}{*}{$\begin{array}{l}\text { Rosenstock } \\
\text { J et al., } 2021 \text { [17] }\end{array}$} & \multirow{4}{*}{$\begin{array}{l}\text { NCT03954834 } \\
\text { (SURPASS-1) }\end{array}$} & \multirow{4}{*}{$\begin{array}{c}\text { India, } \\
\text { Japan, } \\
\text { Mexico, } \\
\text { and USA }\end{array}$} & \multirow{4}{*}{$\begin{array}{l}\text { Multicenter, } \\
\text { double-blind, } \\
\text { randomized, } \\
\text { placebo- } \\
\text { controlled, } \\
\text { phase } 3 \text { trial }\end{array}$} & \multirow{4}{*}{$\begin{array}{l}\text { T2DM ([HbA1c] } \\
\text { 7.0-9.5\%) that was } \\
\text { inadequately } \\
\text { controlled with diet } \\
\text { and exercise alone. } \\
\text { They were naive to } \\
\text { injectable } \\
\text { diabetes therapy. }\end{array}$} & \multirow{4}{*}{4.7} & \multirow{4}{*}{$\begin{array}{l}\text { Change } \\
\text { in HbA1c }\end{array}$} & \multirow{4}{*}{40} & $\begin{array}{l}\text { Tirzepatide } \\
5 \mathrm{mg} / \text { day }\end{array}$ & NR & 121 & 54.1 & 46 & -1.87 \\
\hline & & & & & & & & $\begin{array}{l}\text { Tirzepatide } \\
10 \mathrm{mg} / \text { day }\end{array}$ & $\mathrm{NR}$ & 121 & 55.8 & 60 & -1.89 \\
\hline & & & & & & & & $\begin{array}{l}\text { Tirzepatide } \\
15 \text { mg/day }\end{array}$ & NR & 121 & 52.9 & 52 & -2.07 \\
\hline & & & & & & & & Placebo & NR & 115 & 53.6 & 49 & 0.04 \\
\hline \multirow{5}{*}{$\begin{array}{l}\text { Frias JP et al., } \\
2018 \text { [18] }\end{array}$} & \multirow{5}{*}{ NCT03131687 } & \multirow{5}{*}{$\begin{array}{l}\text { Poland, Puerto } \\
\text { Rico, Slovakia, } \\
\text { and USA }\end{array}$} & \multirow{5}{*}{$\begin{array}{l}\text { Multicenter, phase } \\
2 \mathrm{~b} \text {, randomized, } \\
\text { double- } \\
\text { blind study }\end{array}$} & \multirow{5}{*}{$\begin{array}{l}\text { T2DM for at least } \\
12 \text { months ([HbA1c] } \\
7.0-10.5 \% \text { ) that was } \\
\text { inadequately } \\
\text { controlled with diet } \\
\text { and exercise alone or } \\
\text { with stable } \\
\text { metformin therapy } \\
\text { for at least } 3 \text { months } \\
\text { before screening. }\end{array}$} & \multirow{5}{*}{9.0} & \multirow{5}{*}{$\begin{array}{l}\text { Change } \\
\text { in HbAlc }\end{array}$} & \multirow{5}{*}{26} & $\begin{array}{l}\text { Tirzepatide } \\
1 \mathrm{mg} / \text { day }\end{array}$ & 88.5 & 52 & 57.4 & 56 & -0.7 \\
\hline & & & & & & & & $\begin{array}{l}\text { Tirzepatide } \\
5 \mathrm{mg} / \text { day }\end{array}$ & 89.1 & 55 & 57.9 & 62 & -1.6 \\
\hline & & & & & & & & $\begin{array}{l}\text { Tirzepatide } \\
10 \mathrm{mg} / \text { day }\end{array}$ & 86.3 & 51 & 56.5 & 59 & -2.0 \\
\hline & & & & & & & & $\begin{array}{l}\text { Tirzepatide } \\
15 \mathrm{mg} / \text { day }\end{array}$ & 96.2 & 53 & 56.0 & 42 & -2.4 \\
\hline & & & & & & & & $\begin{array}{l}\text { Dulaglutide } \\
1.5 \mathrm{mg} / \text { day }\end{array}$ & 88.1 & 54 & 58.7 & 44 & -1.1 \\
\hline \multirow{4}{*}{$\begin{array}{l}\text { Frias JP et al., } \\
2020 \text { [19] }\end{array}$} & \multirow{4}{*}{$\begin{array}{l}\text { NCT03311724 } \\
\text { (SURPASS) }\end{array}$} & \multirow{4}{*}{ USA } & \multirow{4}{*}{$\begin{array}{l}\text { Multicenter, phase } \\
\text { 2, randomized, } \\
\text { double-blind, } \\
\text { placebo-controlled }\end{array}$} & \multirow{4}{*}{$\begin{array}{l}\text { T2DM for at least } \\
6 \text { months ([HbA1c] } \\
7.0-10.5 \% \text { ) that was } \\
\text { inadequately } \\
\text { controlled with diet } \\
\text { and exercise alone or } \\
\text { with stable } \\
\text { metformin therapy. }\end{array}$} & \multirow{4}{*}{9.1} & \multirow{4}{*}{$\begin{array}{c}\text { Change } \\
\text { in HbA1c }\end{array}$} & \multirow{4}{*}{12} & $\begin{array}{l}\text { Tirzepatide } \\
12 \text { mg/day }\end{array}$ & 86.2 & 29 & 61.2 & 51.7 & -1.7 \\
\hline & & & & & & & & $\begin{array}{c}\text { Tirzepatide } \\
15 \mathrm{mg} / \text { day-1 }\end{array}$ & 89.3 & 28 & 55.5 & 57.1 & -2 \\
\hline & & & & & & & & $\begin{array}{c}\text { Tirzepatide } \\
15 \mathrm{mg} / \text { day-2 }\end{array}$ & 82.1 & 28 & 56.6 & 82.1 & -1.8 \\
\hline & & & & & & & & Placebo & 88.5 & 26 & 56 & 46.2 & 0.2 \\
\hline \multirow{4}{*}{$\begin{array}{l}\text { Frias JP et al., } \\
2021[20]\end{array}$} & \multirow{4}{*}{$\begin{array}{l}\text { NCT03987919 } \\
\text { (SURPASS-2) }\end{array}$} & \multirow{4}{*}{$\begin{array}{l}\text { USA, UK, } \\
\text { Argentina, } \\
\text { Australia, } \\
\text { Brazil, } \\
\text { Canada, } \\
\text { Israel, Mexico }\end{array}$} & \multirow{4}{*}{$\begin{array}{l}\text { Multicenter, phase } \\
\text { 3, open-label, } \\
\text { parallel-group, } \\
\text { randomized, } \\
\text { active-controlled }\end{array}$} & \multirow{4}{*}{$\begin{array}{l}\text { T2DM for at least } \\
6 \text { months ([HbA1c] } \\
7.0-10.5 \% \text { ) that was } \\
\text { inadequately } \\
\text { controlled with } \\
\text { metformin therapy. }\end{array}$} & \multirow{4}{*}{8.6} & \multirow{4}{*}{$\begin{array}{l}\text { Change } \\
\text { in HbA1c }\end{array}$} & \multirow{4}{*}{40} & $\begin{array}{l}\text { Tirzepatide } \\
5 \mathrm{mg} / \text { day }\end{array}$ & 100 & 470 & 56.3 & 43.6 & -2.01 \\
\hline & & & & & & & & $\begin{array}{l}\text { Tirzepatide } \\
10 \mathrm{mg} / \text { day }\end{array}$ & 100 & 469 & 57.2 & 50.7 & -2.24 \\
\hline & & & & & & & & $\begin{array}{l}\text { Tirzepatide } \\
15 \mathrm{mg} / \text { day }\end{array}$ & 100 & 470 & 55.9 & 45.5 & -2.3 \\
\hline & & & & & & & & $\begin{array}{l}\text { Semaglutide } \\
1 \mathrm{mg} / \text { day }\end{array}$ & 100 & 469 & 56.9 & 48 & -1.86 \\
\hline
\end{tabular}

NR: Not reported; HbA1c: Glycated hemoglobin; T2DM: Type 2 diabetes mellitus; UK: United Kingdom; USA: United States of America. 


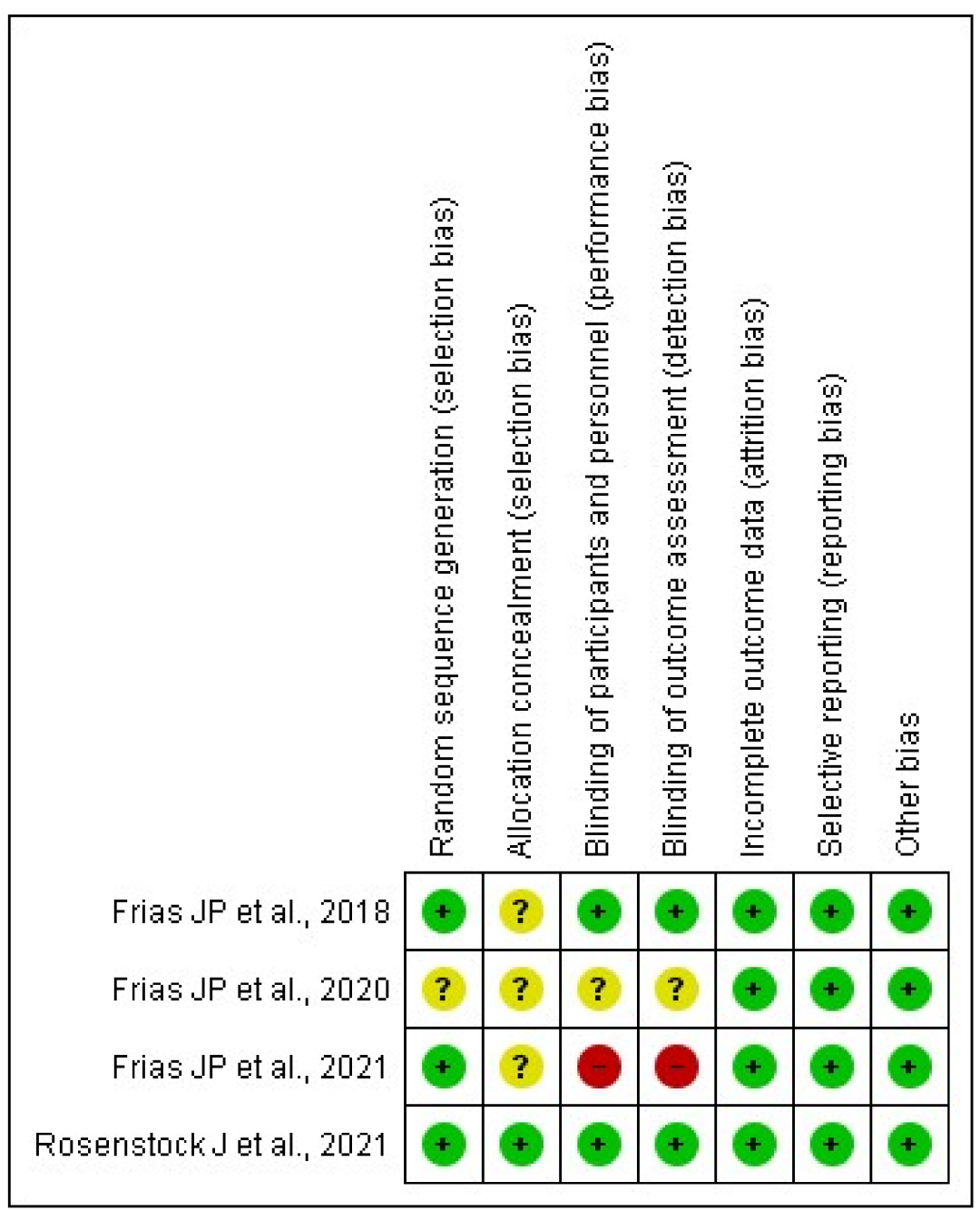

Figure 2. Assessment of the risk of bias in included studies with Cochrane domain-based quality assessment tool.

\subsection{Meta-Analyses}

3.2.1. Efficacy of Tirzepatide on Glycated Hemoglobin

Data on $\mathrm{HbA} 1 \mathrm{c}$ were available from all RCTs. Figure 3 shows the efficacy of different doses of tirzepatide monotherapy or add-on to metformin on $\mathrm{HbA1c}$ using a fixed-effects model. The included studies displayed varying degrees of glycemic efficacy. Overall, metaanalyses showed a significant reduction in $\mathrm{HbA} 1 \mathrm{c}$ versus placebo/controls by $-1.9 \%(95 \%$ CI: -2.0 to $\left.-1.8, p<0.001 ; I^{2}=39.3 \%\right)$, from $-1.8 \%(95 \%$ CI: -1.9 to -1.6$)$ for tirzepatide $5 \mathrm{mg},-1.9 \%$ (95\% CI: -2.1 to -1.8$)$ for $10 \mathrm{mg}$, and $-2.1 \%(95 \% \mathrm{CI}:-2.2$ to -1.9$)$ for $15 \mathrm{mg}$, respectively.

Studies were stratified based on the duration of intervention and showed a more significant and consistent reduction in the $\mathrm{HbA} 1 \mathrm{c}$ after 12 weeks $(-1.9 \%$ (95\% CI: -2.0 to -1.8$)$, 26 weeks $(-1.9 \%, 95 \%$ CI: -2.1 to -1.7$)$, and 40 weeks ( $-1.9 \%$ (95\% CI: -2.0 to -1.8 ) of intervention with tirzepatide than the placebo or controls. More details can be found in Figure 4. 


\section{Tirzepatide, $5 \mathrm{mg}$}

Frias JP et al., 2021

Rosenstock J et al., 2021

Frias JP et al., 2018

Frias JP et al., 2018

Heterogeneity $I^{2}=15.66 \%, H^{2}=1.19$

Test of $\theta_{i}=\theta_{j} \cdot \mathrm{Q}(3)=3.56, \mathrm{p}=0.31$

\section{Tirzepatide, $10 \mathrm{mg}$}

Frias JP et al., 2021

Rosenstock $J$ et al., 2021

Frias JP et al., 2018

Frias JP et al., 2018

Heterogeneity: $\mathrm{I}^{2}=0.00 \%, \mathrm{H}^{2}=1.00$

Test of $\theta_{i}=\theta_{j}: Q(3)=0.94, p=0.82$

\section{Tirzepatide, $15 \mathrm{mg}$}

Frias JP et al., 2021

Rosenstock J et al., 2021

Frias JP et al., 2020

Frias JP et al., 2020

Frias JP et al., 2018

Frias JP et al., 2018

Heterogeneity $\mathrm{I}^{2}=19.06 \%, \mathrm{H}^{2}=1.24$

Test of $\theta_{i}=\theta_{j} \cdot \mathrm{Q}(5)=6.18, \mathrm{p}=0.29$

\section{Overall}

Heterogeneity: $\mathrm{I}^{2}=39.32 \%, \mathrm{H}^{2}=1.65$

Test of $\theta_{i}=\theta_{j}: Q(13)=21.42, p=0.06$

Test of group differences: $\mathrm{Q}_{\mathrm{b}}(2)=10.75, \mathbf{p}<\mathbf{0 . 0 0 1}$

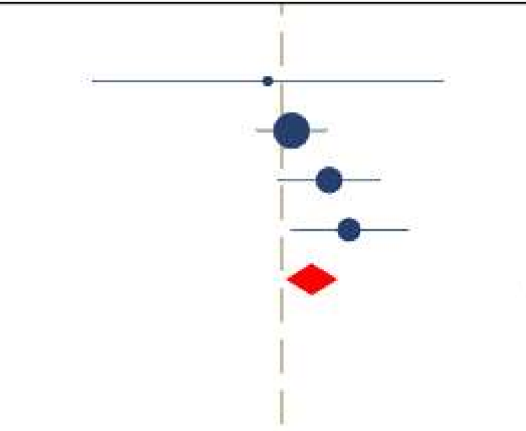

$\begin{array}{rr}-2.01[-2.89,-1.13] & 0.73 \\ -1.89[-2.07,-1.71] & 18.36 \\ -1.70[-1.95,-1.45] & 8.80 \\ -1.60[-1.89,-1.31] & 6.61 \\ -1.79[-1.92,-1.66] & \end{array}$

$\begin{array}{rrr}-2.24[-3.22,-1.26] & 0.60 \\ -1.89[-2.09,-1.69] & 14.88 \\ -2.00[-2.25,-1.75] & 8.80 \\ -2.00[-2.31,-1.69] & 5.81 \\ -1.95[-2.09,-1.81] & \end{array}$

$\begin{array}{llr}-2.30[-3.08, & -1.52] & 0.93 \\ -2.07[-2.27, & -1.87] & 14.88 \\ -2.00[-2.39, & -1.61] & 3.72 \\ -1.80[-2.17, & -1.43] & 4.12 \\ -2.10[-2.39, & -1.81] & 6.61 \\ -2.40[-2.73, & -2.07] & 5.15\end{array}$

$-2.09[-2.22,-1.96]$

$-1.94[-2.02,-1.87]$

Fixed-effects inverse-variance model

Figure 3. Effect of once-weekly tirzepatide on glycated hemoglobin (HbA1c).

\subsubsection{Efficacy of Tirzepatide on Fasting Serum Glucose}

Values of FSG were available from three RCTs. The meta-analysis of available data showed a significant reduction in FSG of $-54.7 \mathrm{mg} / \mathrm{dL}$ (95\% CI: -62.0 to -47.4 ) versus placebo/controls for three different doses of the tirzepatide intervention group, from $-43.6 \mathrm{mg} / \mathrm{dL}(95 \% \mathrm{CI}:-50.2$ to -36.9$)$ for tirzepatide $5 \mathrm{mg},-52.3 \mathrm{mg} / \mathrm{dL}(95 \%$ CI: -66.7 to -37.9$)$ for tirzepatide $10 \mathrm{mg}$, and superior reduction in tirzepatide $15 \mathrm{mg}$, $-61.1 \mathrm{mg} / \mathrm{dL}$ (95\% CI: -73.4 to -48.8 ) was observed (Figure 5). However, the heterogeneity among these studies was statistically significant $\left(I^{2}=72.7 \%, p<0.001\right)$. 


\section{Study}

\section{Week 12}

Frias JP et al., 2018

Frias JP et al. 2018

Frias JP et al., 2020

Frias JP et al., 2018

Heterogeneity: $\mathrm{I}^{2}=37.85 \%, \mathrm{H}^{2}=1.61$

Test of $\theta_{i}=\theta_{i} \cdot \mathrm{Q}(3)=4.83, \mathrm{p}=0.18$

\section{Week 26}

Frias JP et al., 2018

Frias JP et al., 2018

Frias JP et al., 2020

Frias JP et al., 2018

Heterogeneity: $\mathrm{I}^{2}=77.17 \%, \mathrm{H}^{2}=4.38$

Test of $\theta_{i}=\theta_{j}: Q(3)=13.14, p=0.00$

\section{Week 40}

Frias JP et al., 2021

Rosenstock J et al., 2021

Frias JP et al., 2021

Rosenstock J et al., 2021

Frias JP et al., 2021

Rosenstock J et al., 2021

Heterogeneity: $\mathrm{I}^{2}=0.00 \%, \mathrm{H}^{2}=1.00$

Test of $\theta_{i}=\theta_{i}: Q(5)=3.35, p=0.65$

\section{Overall}

Heterogeneity: $\mathrm{I}^{2}=39.32 \%, \mathrm{H}^{2}=1.65$

Test of $\theta_{i}=\theta_{j}: Q(13)=21.42, p=0.06$

Test of group differences:

$Q_{b}(2)=0.11, p=0.95$

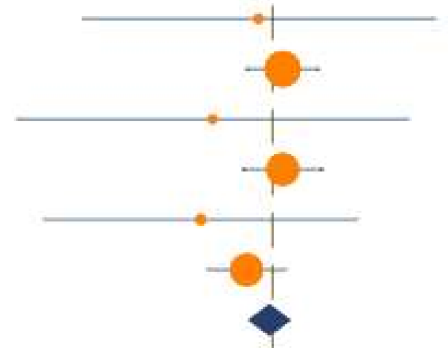

$-2.01\left[\begin{array}{ll}-2.89, & -1.13]\end{array}\right.$

0.73

$-1.89[-2.07,-1.71] \quad 18.36$

$-2.24[-3.22,-1.26]$

0.60

$-1.89\left[\begin{array}{ll}-2.09, & -1.69] \quad 14.88\end{array}\right.$

$-2.30[-3.08,-1.52] \quad 0.93$

$-2.07[-2.27,-1.87] \quad 14.88$

$-1.96[-2.06,-1.85]$

$-1.94[-2.02,-1.87]$

Fixed-effects inverse-variance model

Figure 4. Efficacy of once-weekly tirzepatide on glycated hemoglobin (HbA1c) based on duration of intervention.

\subsubsection{Effect of Tirzepatide on Body Weight}

Data on body weight were available from four RCTs. Random-effects meta-analyses showed a significant reduction in body weight in the tirzepatide group versus the placebo/ controls by $-8.4 \mathrm{~kg}$ (95\% CI: -9.6 to -7.2$)$, from $-7.0 \mathrm{~kg}(95 \% \mathrm{CI}:-7.9$ to -6.0$)$ for tirzepatide $5 \mathrm{mg},-8.6 \mathrm{~kg}(95 \% \mathrm{CI}:-9.6$ to -7.6$)$ for tirzepatide $10 \mathrm{mg}$ and $15 \mathrm{mg}(-8.6 \mathrm{~kg}$, $95 \%$ CI: -10.9 to -6.3 ), respectively (Figure 6 ). There was a considerable heterogeneity among the studies $\left(I^{2}=90.1 \%, p<0.001\right)$. 


\section{Study}

Changes in FSG $(\mathrm{mg} / \mathrm{dL})$ Weight

with $95 \% \mathrm{Cl}$

Tirzepatide, $5 \mathrm{mg}$

Rosenstock J et al., 2021

Heterogeneity: $\mathrm{T}^{2}=0.00, \mathrm{I}^{2}=. \%, \mathrm{H}^{2}=$.

$-43.60[-50.26,-36.94]$

18.36

Test of $\theta_{i}=\theta_{j}: Q(0)=0.00, p=$.

\section{Tirzepatide, $10 \mathrm{mg}$}

Rosenstock J et al., 2021

Frias JP et al., 2018

Heterogeneity: $\mathrm{T}^{2}=83.17, \mathrm{I}^{2}=75.94 \%, \mathrm{H}^{2}=4.16$

Test of $\theta_{i}=\theta_{j}: Q(1)=4.16, p=0.04$

\section{Tirzepatide, $15 \mathrm{mg}$}

Rosenstock J et al., 2021

Frias JP et al., 2020

Frias JP et al., 2020

Frias JP et al., 2018

Heterogeneity: $\mathrm{T}^{2}=108.94, \mathrm{I}^{2}=71.53 \%, \mathrm{H}^{2}=3.51$

Test of $\theta_{i}=\theta_{j}: Q(3)=10.54, p=0.01$

\section{Overall}

Heterogeneity: $\mathrm{T}^{2}=64.58, \mathrm{I}^{2}=72.78 \%, \mathrm{H}^{2}=3.67$

Test of $\theta_{i}=\theta_{j}: Q(6)=22.05, p=0.00$

Test of group differences: $Q_{b}(2)=6.39, \mathbf{p}=\mathbf{0 . 0 4}$

Random-effects DerSimonian-Laird model

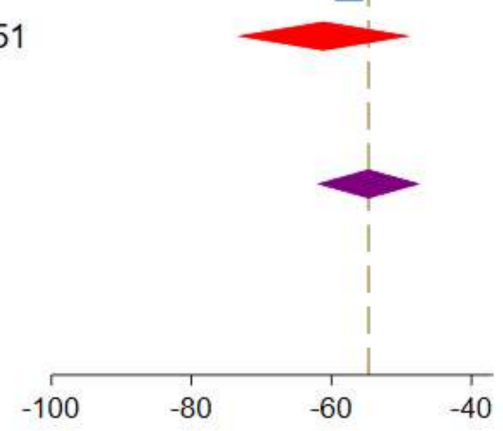

$-43.60[-50.26,-36.94]$

$-45.90[-52.76,-39.04]$

Figure 5. Effect of once-weekly tirzepatide on fasting serum glucose levels (mg/dL).

\subsubsection{Overall Safety}

Table 2 shows the proportion of representative adverse events in the tirzepatidetreated group. All the studies provided data on the safety outcomes. The overall pooled proportion of hypoglycemic events was 3\% (95\% CI: 2-5), 4\% (95\% CI: 2-6) for serious AEs, and AEs leading to discontinuation of therapy were 7\% (95\% CI: 5-8). A significantly higher proportion of patients experienced gastrointestinal adverse events with tirzepatide (44\%, 95\% CI: 40-48).

\subsubsection{Publication Bias}

A visual inspection of funnel plots for the primary outcome (HbA1c) suggested an absence of publication bias and of a small study effect, the Egger's test $(p=0.289)$ and Begg's test $(p=0.620)$ indicated no evidence of publication bias (Figure 7). Because a publication bias assessment was not recommended for less than 10 pooled studies, we did not assess the existence of publication on FSG and body weight. 
Study

Changes in bodyweight Weight with $95 \% \mathrm{Cl}$

(\%)

\section{Tirzepatide, $\mathbf{5 m g}$}

Rosenstock J et al., 2021

Heterogeneity: $\mathrm{T}^{2}=0.00, \mathrm{I}^{2}=\%, \mathrm{H}^{2}=$.

Test of $\theta_{i}=\theta_{j}: Q(0)=0.00, p=$.

\section{Tirzepatide, $10 \mathrm{mg}$}

Rosenstock J et al., 2021

Frias JP et al., 2018

Frias JP et al., 2021

Heterogeneity: $\mathrm{T}^{2}=0.54, \mathrm{I}^{2}=66.98 \%, \mathrm{H}^{2}=3.03$

Test of $\theta_{i}=\theta_{\mathrm{j}}: \mathrm{Q}(2)=6.06, \mathrm{p}=0.05$

\section{Tirzepatide, $15 \mathrm{mg}$}

Rosenstock J et al., 2021

Frias JP et al., 2021

Frias JP et al., 2020

Frias JP et al., 2020

Frias JP et al., 2018

Heterogeneity: $\mathrm{T}^{2}=6.31, \mathrm{I}^{2}=93.42 \%_{\mathrm{s}} \mathrm{H}^{2}=15.19$

Test of $\theta_{\mathrm{i}}=\theta_{\mathrm{j}}: \mathrm{Q}(4)=60.76, \mathrm{p}=0.00$

\section{Overall}

Heterogeneity: $\mathrm{T}^{2}=2.91, \mathrm{I}^{2}=90.11 \%, \mathrm{H}^{2}=10.11$

Test of $\theta_{\mathrm{i}}=\theta_{\mathrm{j}}: \mathrm{Q}(8)=80.88, \mathrm{p}=0.00$

Test of group differences: $Q_{b}(2)=5.62, \mathbf{p}=\mathbf{0 . 0 6}$

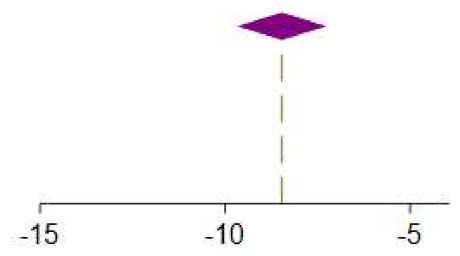

$-7.00[-7.98,-6.02] \quad 11.68$

$-7.00[-7.98,-6.02]$

$-7.80[-8.78,-6.82]$

$-8.70[-10.27,-7.13]$

$-9.30[-9.99,-8.61]$

11.68

10.40

12.17

$-8.64[-9.66,-7.61]$

$-9.50[-10.48,-8.52]$

11.68

$-11.20[-12.26,-10.14]$

11.53

10.40

10.44

$-5.70[-7.25,-4.15]$

10.02
$-8.47[-9.66,-7.27]$

Random-effects DerSimonian-Laird model

Figure 6. Effect of once-weekly tirzepatide on body weight (kg).

Table 2. Safety of tirzepatide.

\begin{tabular}{|c|c|c|c|c|c|c|}
\hline \multirow[b]{2}{*}{ Events } & \multicolumn{4}{|c|}{ Proportion $(95 \% \mathrm{CI})$} & \multirow[b]{2}{*}{$I^{2}$} & \multirow[b]{2}{*}{$P_{\text {hetetogeneity }}$} \\
\hline & Total & $\begin{array}{l}\text { Tirzepatide, } \\
5 \mathrm{mg}\end{array}$ & $\begin{array}{l}\text { Tirzepatide, } \\
10 \mathrm{mg}\end{array}$ & $\begin{array}{l}\text { Tirzepatide, } \\
15 \mathrm{mg}\end{array}$ & & \\
\hline Total adverse events & $70(67-74)$ & $66(61-71)$ & $70(65-75)$ & $73(65-81)$ & $58.5 \%$ & 0.305 \\
\hline Serious adverse events & $4(2-6)$ & $5(1-8)$ & $4(1-7)$ & $3(0-7)$ & $73.2 \%$ & 0.071 \\
\hline Hypoglycaemia & $3(2-5)$ & $4(1-8)$ & $5(1-11)$ & $7(2-13)$ & $80.1 \%$ & 0.594 \\
\hline All gastrointestinal events & $44(40-48)$ & $39(35-43)$ & $46(42-49)$ & $50(38-61)$ & $63.6 \%$ & 0.025 \\
\hline Nausea & $5(2-7)$ & $1(0-2)$ & $3(0-7)$ & $12(6-18)$ & $89.9 \%$ & $<0.001$ \\
\hline Diarrhea & $3(2-5)$ & $0(0-1)$ & $3(1-8)$ & $13(6-19)$ & $89.9 \%$ & $<0.001$ \\
\hline $\begin{array}{l}\text { Adverse events leading to } \\
\text { discontinuation of therapy }\end{array}$ & $7(5-8)$ & $5(3-8)$ & $7(5-10)$ & $8(4-12)$ & $51.7 \%$ & 0.441 \\
\hline
\end{tabular}




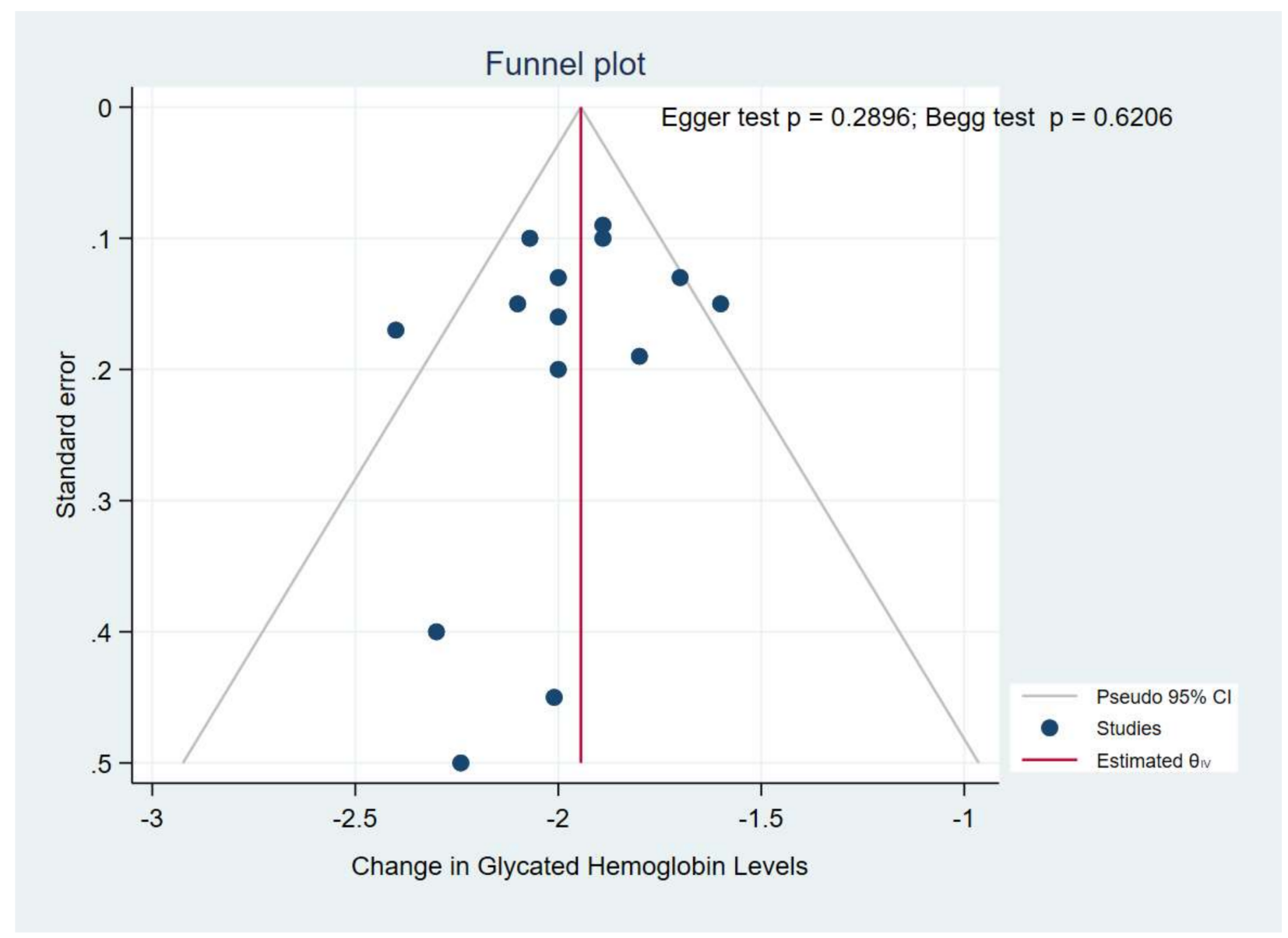

Figure 7. Funnel plot.

\section{Discussion}

T2DM is a chronic disease characterized by hyperglycemia and progressive dysregulation of the insulin-glucose feedback mechanism, and glucose-lowering is a mainstay of treatment [21]. Novel glucose-lowering drugs such as selective GLP-1 receptor agonists, especially once-weekly dulaglutide and semaglutide, showed a substantial improvement in glucose control and promoted weight loss [22]. Despite these advances in therapy for the management of hyperglycemia, many people with T2DM did not achieve optimal glycemic control. Notably, the co-administration of the GLP-1 receptor agonist and GIP in mice with T2DM and obesity showed a greater improvement in glycemic and body weight than with selective GLP-1 receptor agonists [23]. This rationale paved the way to target both incretin hormones and the development of a dual GLP-1/GIP receptor agonist, referred to as a "twincretin" for the treatment of T2DM [9]. Tirzepatide is a novel therapeutic agent administered once a week as a dual GLP-1/GIP receptor, formulated as a 39-amino acid synthetic peptide, based on the native GIP sequence [9]. In individual RCTs, tirzepatide alone or added to metformin was shown to improve glycemic control with weight loss compared with a placebo or selective GLP-1 receptor agonists (dulaglutide or semaglutide) [17-20]. However, to date, no systematic compilation of the available evidence of tirzepatide has been performed.

We presented the relative efficacy and safety of tirzepatide monotherapy or add-on to metformin compared with the placebo or selective GLP-1 receptor agonists to provide a comprehensive analysis of the tirzepatide. Compared to the placebo or the selective GLP-1 receptor agonists, tirzepatide improved glycemic control $(-2.0$ to $-1.8 \%$ in $\mathrm{HbA1c}$ and -62.0 to $-47.3 \mathrm{mg} / \mathrm{dL}$ in FSG) and reduced body weight ( -9.6 to $-7.2 \mathrm{~kg}$ ). All three tirzepatide doses showed significant $\mathrm{HbA} 1 \mathrm{c}$ reductions, and the robust glycemic effect was 
consistently maintained from week 12 to week 40 . Available evidence from the published RCTs also suggested a greater decrease in HbA1c, FSG, and body weight [19-22]. Given the limited data available for postprandial self-monitored blood glucose profile, however, the effect of tirzepatide on the achievement of the guidelines' recommended $\mathrm{HbA} 1 \mathrm{c}$ targets and weight loss of $5 \%$ or greater should be further investigated. Overall, the highest dose of tirzepatide had superior effects on $\mathrm{HbA} 1 \mathrm{c}$ reduction, and the change in the $\mathrm{HbA} 1 \mathrm{c}$ presumably occurred as early as 12 weeks, with no differences noted at week 26 or 40 . The most recent phase 3 trial (SURPASS-1) has indeed demonstrated a similar reduction in $\mathrm{HbA1c}$, FSG, and body weight in patients with T2DM treated with tirzepatide $(5,10$, and $15 \mathrm{mg}$ ) as monotherapy [17]. Ongoing RCTs [24-29] will confirm whether similar benefits could be expected in an overweight or obese population with (SURMOUNT-2) [29] or without T2DM (SURMOUNT-3, SURMOUNT-4) $[25,26]$ and in patients with T2DM inadequately controlled on insulin glargine with/without metformin (SURPASS-6) [27]. Moreover, a recently completed phase 1 trial on tirzepatide (NCT03951753) may provide further evidence on the relationship between $\mathrm{HbA} 1 \mathrm{c}$ and body weight reduction in patients with T2DM [28].

Along with changes in $\mathrm{HbA1c}$, we also found some marginal differences with tirzepatide doses on FSG and body weight. The highest dose of tirzepatide $(15 \mathrm{mg})$ reduced FSG to a greater extent than other doses $(5 \mathrm{mg}$ and $10 \mathrm{mg}$ ). Conversely, tirzepatide $10 \mathrm{mg}$ and $15 \mathrm{mg}$ did not differ in the extent of body weight reduction. Little data on FSG and body weight with different doses of tirzepatide are reported in the included RCTs, limiting the ability to investigate clinically meaningful differences between higher and lower doses and also accounting for different background therapies. Indeed, the differences observed for some clinical outcomes could be attributed to the intrinsic pharmacological properties of investigational drugs at different doses.

As for safety, tirzepatide therapy did not lead to a higher incidence of all AEs than the placebo or selective GLP-1 receptor agonists, and individual studies indicated that most AEs were mild or moderate in severity [17-20]. The most prevalent AEs were gastrointestinal (44\%), including nausea and diarrhea, and similar AEs observed in current GLP-1 analogs [30]. Moreover, a limited proportion of participants experienced hypoglycemia $(3 \%)$ and AEs leading to the discontinuation of tirzepatide (7\%), most of which are dosedependent incidences. Ongoing RCTs [5-7,9] will confirm whether tirzepatide exhibits long-term safety, and SURPASS-CVOT (NCT04255433) [31] will provide more data on the cardiovascular safety of tirzepatide compared with dulaglutide.

To the best of our knowledge, this is the first systematic review and meta-analysis that combined the data of all tirzepatide phase 2 and phase 3 RCTs with a mixed-treatment comparison meta-analytical approach aiming to provide a comprehensive picture of clinically meaningful efficacy and safety outcomes. However, we should acknowledge some limitations of this study. We identified a small number of studies and performed a metaanalysis based only on data published in journal articles or available at ClinicalTrials.gov. Given the limited available data, we pooled efficacy data for HbA1c, FSG, and body weight and could not perform a subgroup analysis to investigate the source of heterogeneity for some outcomes. However, RCTs, ethnic differences in the population, differences in the settings, follow-up duration or outcome selections, definitions, and ascertainment could differ. Moreover, we found a data inconsistency for the FSG and body weight meta-analysis, and caution is recommended in interpreting these results. Lastly, the current duration of studies was very limited, and data about tirzepatide in the long term were deficient.

\section{Conclusions}

Tirzepatide, a novel incretin-based therapy for T2DM, showed robust $\mathrm{HbA1c}(-1.94 \%)$, FSG $(-54.7 \mathrm{mg} / \mathrm{dl})$, and body weight $(-8.5 \mathrm{~kg})$ reductions, without an increased risk of hypoglycemia. At the highest dose $(15 \mathrm{mg})$, tirzepatide reduced: HbA1c $(-2.1 \%)$, FSG $(-61.1 \mathrm{mg} / \mathrm{dl})$, and body weight $(-8.6 \mathrm{~kg})$. The study also found that tirzepatide had an acceptable safety profile similar to the GLP-1 receptor agonists for the treatment of T2DM. 
Further research is needed to determine the long-term safety and efficacy of tirzepatide in achieving guideline-recommended cardiometabolic targets.

Author Contributions: A.S.B., K.V. and W.T. contributed equally to the manuscript. All authors have read and agreed to the published version of the manuscript.

Funding: No external source of funding.

Institutional Review Board Statement: Not applicable.

Informed Consent Statement: Not applicable.

Data Availability Statement: Data sharing not applicable.

Conflicts of Interest: The authors declare no conflict of interest.

\section{References}

1. Saeedi, P.; Petersohn, I.; Salpea, P.; Malanda, B.; Karuranga, S.; Unwin, N.; Colagiuri, S.; Guariguata, L.; Motala, A.A.; Ogurtsova, K.; et al. Global and regional diabetes prevalence estimates for 2019 and projections for 2030 and 2045: Results from the International Diabetes Federation Diabetes Atlas, 9th edition. Diabetes Res. Clin. Pract. 2019, 157, 7843. [CrossRef] [PubMed]

2. Gerstein, H.; Pogue, J.; Mann, J.; Lonn, E.; Dagenais, G.; McQueen, M.; Yusuf, S. The relationship between dysglycaemia and cardiovascular and renal risk in diabetic and non-diabetic participants in the HOPE study: A prospective epidemiological analysis. Diabetologia 2005, 48, 1749-1755. [CrossRef]

3. Bailes, B.K. Diabetes mellitus and its chronic complications. AORN J. 2002, 76, 265-282. [CrossRef]

4. Colagiuri, S. Optimal management of type 2 diabetes: The evidence. Diabetes Obes. Metab. 2012, 14, 3-8. [CrossRef]

5. Min, T.; Bain, S.C. The Role of Tirzepatide, Dual GIP and GLP-1 Receptor Agonist, in the Management of Type 2 Diabetes: The SURPASS Clinical Trials. Diabetes Ther. Res. Treat. Educ. Diabetes Relat. Disord. 2021, 12, 143-157. [CrossRef]

6. Palmer, S.C.; Tendal, B.; Mustafa, R.A.; Vandvik, P.O.; Li, S.; Hao, Q.; Tunnicliffe, D.; Ruospo, M.; Natale, P.; Saglimbene, V.; et al. Sodium-glucose cotransporter protein-2 (SGLT-2) inhibitors and glucagon-like peptide-1 (GLP-1) receptor agonists for type 2 diabetes: Systematic review and network meta-analysis of randomised controlled trials. BMJ 2021, 372, m4573. [CrossRef]

7. Andersen, A.; Lund, A.; Knop, F.K.; Vilsbøll, T. Glucagon-like peptide 1 in health and disease. Nat. Rev. Endocrinol. 2018, 14, 390-403. [CrossRef]

8. Holst, J.J.; Rosenkilde, M.M. GIP as a Therapeutic Target in Diabetes and Obesity: Insight from Incretin Co-agonists. J. Clin. Endocrinol. Metab. 2020, 105, e2710-e2716. [CrossRef]

9. Coskun, T.; Sloop, K.W.; Loghin, C.; Alsina-Fernandez, J.; Urva, S.; Bokvist, K.B.; Cui, X.; Briere, D.A.; Cabrera, O.; Roell, W.C.; et al. LY3298176, a novel dual GIP and GLP-1 receptor agonist for the treatment of type 2 diabetes mellitus: From discovery to clinical proof of concept. Mol. Metab. 2018, 18, 3-14. [CrossRef]

10. Frías, J.P. Tirzepatide: A glucose-dependent insulinotropic polypeptide (GIP) and glucagon-like peptide-1 (GLP-1) dual agonist in development for the treatment of type 2 diabetes. Expert. Rev. Endocrinol. Metab. 2020, 15, 379-394. [CrossRef]

11. Nauck, M.; Bartels, E.; Orskov, C.; Ebert, R.; Creutzfeldt, W. Additive insulinotropic effects of exogenous synthetic human gastric inhibitory polypeptide and glucagon-like peptide-1-(7-36) amide infused at near-physiological insulinotropic hormone and glucose concentrations. J. Clin. Endocrinol. Metab. 1993, 76, 912-917. [CrossRef] [PubMed]

12. Elahi, D.; McAloon-Dyke, M.; Fukagawa, N.K.; Meneilly, G.S.; Sclater, A.L.; Minaker, K.L.; Habener, J.F.; Andersen, D.K. The insulinotropic actions of glucose-dependent insulinotropic polypeptide (GIP) and glucagon-like peptide-1 (7-37) in normal and diabetic subjects. Regul. Pept. 1994, 51, 63-74. [CrossRef]

13. Page, M.J.; McKenzie, J.E.; Bossuyt, P.M.; Boutron, I.; Hoffmann, T.C.; Mulrow, C.D.; Shamseer, L.; Tetzlaff, J.M.; Akl, E.A.; Brennan, S.E.; et al. The PRISMA 2020 statement: An updated guideline for reporting systematic reviews. BMJ 2021, 372 , n71. [CrossRef] [PubMed]

14. Sterne, J.A.C.; Savovic, J.; Page, M.J.; Elbers, R.G.; Blencowe, N.S.; Boutron, I.; Cates, C.J.; Cheng, H.Y.; Corbett, M.S.; Eldridge, S.M.; et al. RoB 2: A revised tool for assessing risk of bias in randomised trials. BMJ 2019, 366, 14898. [CrossRef]

15. Cumpston, M.; Li, T.; Page, M.J.; Chandler, J.; Welch, V.A.; Higgins, J.P.; Thomas, J. Updated guidance for trusted systematic reviews: A new edition of the Cochrane Handbook for Systematic Reviews of Interventions. Cochrane Database Syst. Rev. 2019, 10, Ed000142. [CrossRef] [PubMed]

16. Higgins, J.; Thomas, J. Cochrane Handbook for Systematic Reviews of Interventions. 2019. Available online: https://training. cochrane.org/handbook/current (accessed on 27 July 2021).

17. Rosenstock, J.; Wysham, C.; Frías, J.P.; Kaneko, S.; Lee, C.J.; Fernández Landó, L.; Mao, H.; Cui, X.; Karanikas, C.A.; Thieu, V.T. Efficacy and safety of a novel dual GIP and GLP-1 receptor agonist tirzepatide in patients with type 2 diabetes (SURPASS-1): A double-blind, randomised, phase 3 trial. Lancet 2021, 398, 143-155. [CrossRef]

18. Frias, J.P.; Nauck, M.A.; Van, J.; Kutner, M.E.; Cui, X.; Benson, C.; Urva, S.; Gimeno, R.E.; Milicevic, Z.; Robins, D.; et al. Efficacy and safety of LY3298176, a novel dual GIP and GLP-1 receptor agonist, in patients with type 2 diabetes: A randomised, placebo-controlled and active comparator-controlled phase 2 trial. Lancet 2018, 392, 2180-2193. [CrossRef] 
19. Frias, J.P.; Nauck, M.A.; Van, J.; Benson, C.; Bray, R.; Cui, X.; Milicevic, Z.; Urva, S.; Haupt, A.; Robins, D.A. Efficacy and tolerability of tirzepatide, a dual glucose-dependent insulinotropic peptide and glucagon-like peptide-1 receptor agonist in patients with type 2 diabetes: A 12-week, randomized, double-blind, placebo-controlled study to evaluate different dose-escalation regimens. Diabetes Obes. Metab. 2020, 22, 938-946. [CrossRef]

20. Frías, J.P.; Davies, M.J.; Rosenstock, J.; Pérez Manghi, F.C.; Fernández Landó, L.; Bergman, B.K.; Liu, B.; Cui, X.; Brown, K. Tirzepatide versus Semaglutide Once Weekly in Patients with Type 2 Diabetes. N. Engl. J. Med. 2021, 385, 503-515. [CrossRef]

21. Pharmacologic Approaches to Glycemic Treatment: Standards of Medical Care in Diabetes-2019. Diabetes Care 2019, 42, S90-S102. [CrossRef]

22. Pharmacologic Approaches to Glycemic Treatment: Standards of Medical Care in Diabetes-2021. Diabetes Care 2021, 44, S111-S124. [CrossRef] [PubMed]

23. Gault, V.A.; Kerr, B.D.; Harriott, P.; Flatt, P.R. Administration of an acylated GLP-1 and GIP preparation provides added beneficial glucose-lowering and insulinotropic actions over single incretins in mice with Type 2 diabetes and obesity. Clin. Sci. 2011, 121, 107-117. [CrossRef] [PubMed]

24. ClinicalTrials.gov. Available online: https://clinicaltrials.gov/ct2/show/NCT04657016 (accessed on 31 August 2021).

25. A Study of Tirzepatide (LY3298176) in Participants with Obesity or Overweight for the Maintenance of Weight Loss (SURMOUNT4). Available online: https: / clinicaltrials.gov/ct2/show /NCT04660643 (accessed on 31 August 2021).

26. A Study of Tirzepatide (LY3298176) In Participants after a Lifestyle Weight Loss Program (SURMOUNT-3). Available online: https:/ / clinicaltrials.gov/ct2/show / NCT04657016 (accessed on 31 August 2021).

27. A Study of Tirzepatide (LY3298176) Versus Insulin Lispro (U100) in Participants with Type 2 Diabetes Inadequately Controlled on Insulin Glargine (U100) with or without Metformin (SURPASS-6). Available online: https:// clinicaltrials.gov/ct2/show/NCT045 37923 (accessed on 31 August 2021).

28. A Study of Tirzepatide in Participants with Type 2 Diabetes Mellitus (T2DM). Available online: https://clinicaltrials.gov/ct2 / show / NCT03951753 (accessed on 31 August 2021).

29. A Study of Tirzepatide (LY3298176) in Participants with Type 2 Diabetes Who Have Obesity or Are Overweight (SURMOUNT-2). Available online: https: / clinicaltrials.gov/ct2/show/NCT04657003 (accessed on 31 August 2021).

30. Sun, F.; Chai, S.; Yu, K.; Quan, X.; Yang, Z.; Wu, S.; Zhang, Y.; Ji, L.; Wang, J.; Shi, L. Gastrointestinal adverse events of glucagonlike peptide-1 receptor agonists in patients with type 2 diabetes: A systematic review and network meta-analysis. Diabetes Technol. Ther. 2015, 17, 35-42. [CrossRef] [PubMed]

31. A Study of Tirzepatide (LY3298176) Compared with Dulaglutide on Major Cardiovascular Events in Participants with Type 2 Diabetes (SURPASS-CVOT). 2020. Available online: https:/ / clinicaltrials.gov/ct2/show / NCT04255433 (accessed on 31 August 2021). 